\title{
A plea for clear language on vaccine safety
}

"If you can't explain it simply, you don't understand it well enough." - Albert Einstein

$\mathrm{M}$ ost academics see their fellow academics as the main audience for their scientific work. But the Web and search engines such as Google have made academic articles and reports widely accessible. This newfound availability can be problematic. The careful, precise academic language that is the backbone of scientific writing - the language of scholarly articles and reports — can lead nonacademics to draw incorrect conclusions about the information being presented. The academic literature about vaccine safety provides some powerful examples of the troubling gap between academic intent and public conclusions.

Immunization against childhood diseases is one of medicine's greatest achievements, having prevented millions of deaths and disabilities. But the very success of vaccination has had unintended consequences. The public has little or no personal knowledge about the consequences of these once terrible vaccine-preventable diseases and now pays far more attention to concerns about vaccine safety. Many of these concerns have been greatly exaggerated. ${ }^{1}$

Although much has been written about the importance of providing information about the risks of diseases and the benefits and safety of vaccines in clear language on consent

Box 1: Conclusion about the relation between the measles-mumps-rubella (MMR) vaccine and autism: The Institute of Medicine's version ${ }^{2}$

"Thus, the committee concludes that the evidence favors rejection of a causal relationship at the population level between MMR vaccine and autistic spectrum disorders (ASD). The committee bases this conclusion on the following evidence:

- A consistent body of epidemiological evidence shows no association at a population level between MMR vaccine and ASD.

- The original case series of children with ASD and bowel symptoms and other available case reports are uninformative with respect to causality.

- Biologic models linking MMR vaccine and ASD are fragmentary.

- There is no relevant animal model linking MMR vaccine and ASD.

However, the committee notes that its conclusion does not exclude the possibility that MMR vaccine could contribute to ASD in a small number of children, because the epidemiological evidence lacks the precision to assess rare occurrences of a response to MMR vaccine leading to ASD and the proposed biological models linking MMR vaccine to ASD, although far from established, are nevertheless not disproved." forms for vaccination, little attention has been paid to the same need for clarity in the language of scientific reports and academic articles. In the wired world, the audience for these articles includes the media, politicians, the general public, vaccine supporters, antivaccine lobbyists and health care workers. The cautious, exact scientific language of academic articles is largely alien to many members of these groups and can obscure the meaning of the scientific findings and fuel misinformation.

The conclusion in the executive summary of the $2001 \mathrm{In}$ stitute of Medicine report about the relation between the measles-mumps-rubella vaccine and autism ${ }^{2}$ provides an excellent example of potentially confusing academic language (Box 1). This conclusion was carefully crafted in precise academic language - so precise that, depending on the reader, different conclusions can be drawn. The academic involved in vaccine research, familiar with the scientific principle that the null hypothesis cannot be proven, would conclude that this report does not find evidence that the measles-mumps-rubella vaccine causes autism. A health care worker reading this same statement may not be sure what to make of it: maybe the vaccine causes autism in some children, just not often. The politician may wonder whether supporting public programs for measles-mumps-rubella vaccination at this time is justified. The journalist may reasonably interpret the conclusion as saying that the vaccine is a cause of autism, albeit an infrequent one. The antivaccine lobby, for its part, would be delighted that this respected academic body has given support to its claims that the vaccine can cause autism. Contrast the statements in Box 1 with those in Box 2, which presents our summary of the same conclusion in clear language.

Unclear meanings are not just an issue for academic articles and reports about vaccine safety. When a serious adverse event follows vaccination, the patient, caregiver, program provider and members of the general public rightfully want to know: Did the vaccine cause the serious event or was this just a coincidence? Rigorous studies to determine whether one event caused another follow a formal scientific process..$^{3-5}$ Once these are completed, the relation between the vaccine and the serious event is reported as very likely or certain, probable, possible, unlikely, unrelated or unclassifiable. ${ }^{4}$ For

\section{Box 2: Conclusion about the relation between the measles-mumps-rubella vaccine and autism: our clear-language version}

A thorough review of the research involving people and animals provides no evidence that the measles-mumpsrubella vaccine causes autism. However, because the causes of autism are unknown, research on autism needs to continue. 
scientific precision, the term "unlikely" is used when there is no evidence that the vaccine caused the event but no firm evidence for another cause. From a scientific perspective, the event cannot be classified as "unrelated," because this is unproven. Ironically, the term "unlikely" may imply the opposite to nonacademics - that there is a causal association between the adverse event and the vaccine administered.

Such serious misunderstandings, whether by health care workers, community members, politicians or journalists, can lead to a widespread community belief that the vaccine in question is unsafe. Instead of calming fears, the misinterpreted conclusion may add to anxiety. The vaccine program may be stopped as a result, leading to a rise in the incidence of the disease and, in some instances, deaths and disabilities that could have been prevented by vaccination. Some developing countries have made a plea that the outcomes of vaccine causality assessments be reported in clear language that cannot be misinterpreted (i.e., a statement that the vaccine either did or did not cause the adverse event).

The harms that can occur when academic language obscures meaning are serious and will only increase if we do not improve our communication skills. Academic jargon that obscures meaning must be replaced by crisp, understandable conclusions. We know that this will not be easy. All medical academics should receive formal education about how to write in a clear and straightforward manner and why this is important. We urge all academic writers, medical journal editors and reviewers of academic reports to ensure that the meaning of an article's conclusion or a report's summary is clear and cannot be misinterpreted.

Noni MacDonald MD MSc

Section Editor, Public Health

CMAJ

André Picard

Public health reporter

The Globe and Mail

With the Editorial-Writing Team (Paul C. Hébert MD MHSc, Matthew B. Stanbrook MD PhD and Ken Flegel MDCM MSc)

Competing interests: See www.cmaj.ca/misc/edboard.shtml for the EditorialWriting Team's statements. None declared for André Picard.

\section{REFERENCES}

1. Fisher MC. Vaccine safety. Pediatr Infect Dis J 2008;27:827-30.

2. Stratton K, Gable A, Shetty P, et al., editors; Institute of Medicine, Board on Health Promotion and Disease Prevention, Immunization Safety Review Committee. Immunization safety review: measles-mumps-rubella vaccine and autism. Washington (DC): National Academies Press; 2001.

3. World Health Organization Global Advisory Committee on Vaccine Safety. Causality assessment of adverse events following immunization. Wkly Epidemiol Rec 2001;76:85-9.

4. World Health Organization. Adverse events following immunization (AEFI): causality assessment. Aide memoire. Geneva (Switzerland): The Organization. Available: www.who.int/vaccines-documents/DocsPDF05/815.pdf (accessed 2009 Feb. 25).

5. Collet JP, MacDonald N, Cashman N, et al.; Advisory Committee on Causality Assessment. Monitoring signals for vaccine safety: the assessment of individual adverse event reports by an expert advisory committee. Bull World Health Organ 2000;78:178-85. 